\title{
Financiando esfuerzos contra el cambio climático en américa latina
} Afinancing efforts against climate change in latin america

\author{
Sandra, Guzmán ${ }^{1}$ \\ Mariana, Castillo \\ Alin, Moncada ${ }^{3}$
}

Grupo de Financiamiento Climático para América Latina y el Caribe (GFLAC)

\begin{abstract}
RESUMEN
El presente artículo es producto de una revisión bibliográfica, cuyo objetivo consistió en presentar una visión sobre la evolución del financiamiento climático a nivel global, y cómo éste ha impactado en la región de América Latina, con particular énfasis en la participación del financiamiento en materia de energías alternativas y renovables. Se aplicó el método de análisis, con un enfoque cualitativo, diseño no experimental, bajo un nivel documental-bibliográfico transversal. Tras la revisión documental se realiza un breve análisis de cómo se ubica México en estas discusiones y las necesidades nacionales que se derivan de los procesos internacionales como el Acuerdo de París. Se concluye que se requiere una acción conjunta, coordinada y determinante de todos los actores involucrados. Los gobiernos nacionales y locales, el sector privado, los fondos multilaterales, la banca, la sociedad civil, etc.
\end{abstract}

Palabras clave: América Latina, cambio climático, desarrollo sustentable, y financiamiento.

\begin{abstract}
This article is the product of a literature review, whose aim consisted in submitting a vision about the evolution of the funding of climate change in a global level, and how this has had an impact on the Latin America region, with a special focus on the funding participation with regard to alternative and renewable energies. The method of analysis was applied with a qualitative approach, non-experimental design, under a documentary-literature cross sectional level. After the documentary review a brief analysis is done about where Mexico is in these arguments and the needs that arise from the international processes such as the Paris Agreement. It concludes that it is necessary a coordinate, determining and joint action from all of the involved actors. The local and national governments, the private sector, multilateral funds, banking, civil society, etc.
\end{abstract}

Key words: Latin America, Climate Change, Sustainable Development, Funding.

\section{Cómo referenciar este artículo:}

Guzmán, S., Castillo, M., \& Moncada, A. (2017). Financiando esfuerzos contra el cambio climático en américa latina. Política,Globalidad y Ciudadanía, 65-74.

Recibido: 23 de Agosto 2016 - Aceptado: 26 de Octure 2016

(cc) EY-NC-ND

1 Master en Política y Regulación Ambiental por la London School of Economics and Political Sciencie (LSE).

2 Coordinadora de la Agenda Naciaonal, Grupo de Financiamiento Climático de América Latina y el Caribe (GFLAC). alin.moncada@gmail.com

3 Coordinadora de Comunicación Estratégica, Grupo de Financiamiento Climático de Americe Latina y el Caribe (GFLAC). alin.moncada@gmail.com

Revista Política, Globalidad y Ciudadanía, Vol. 3 No. 5, Enero - Junio 2017, Universidad Autónoma de Nuevo León, Monterrey, México Monterrey, México, ISSN 2395-8448. pp 65-77. http://revpoliticas.uanl.mx/index.php/RPGyC/article/view/68 


\section{1.- INTRODUCCIÓN}

Las soluciones a los impactos y consecuencias del cambio climático se han ido constituyendo desde un enfoque multi nivel y multi sector, debido a un creciente reconocimiento de que la acción unilateral, o uni-sectorial no servirán para contrarrestar los impactos hasta ahora ocasionados por el problema.

A nivel global, se han hecho estimaciones sobre el costo del cambio climático y sobre el costo de la inacción (Stern, 2006, 2009). Aunque los montos han variado de estudio a estudio, se calcula que el costo de atender el problema podría representar al menos el $2 \%$ del producto interno del mundo; sin embargo, se habla de que el costo de la inacción podría significar hasta el 20\% (Stern, 2006, 2009).

Estas estimaciones han generado un debate internacional que hoy se ha traducido en una creciente discusión sobre los medios necesarios para financiar las acciones requeridas, con el propósito de reducir las emisiones que causan el problema, así como para financiar acciones que incrementen la resiliencia y disminuyan la vulnerabilidad.

En este capítulo, se presenta una visión sobre la evolución del tema de financiamiento climático a nivel global, y cómo esto ha impactado a la región de América Latina, con un particular énfasis en la participación del financiamiento en materia de energía renovable. De igual forma, se presenta un breve análisis de cómo se ubica México en estas discusiones y las necesidades nacionales que se derivan de los procesos internacionales, como lo es el Acuerdo de París.

\section{2.- FUNDAMENTO TEÓRICO}

\section{Perspectivas del financiamiento climático a nivel global}

El tema de financiamiento ha sido uno de los pilares de las discusiones globales desde la Conferencia de las Naciones Unidas sobre el Medio Humano celebrada en Estocolmo en 1972, en la que ya se hablaba de los costos que la destrucción ambiental podría traer a los países. Estos argumentos sentaron las bases para que el tema de financiamiento se colocara en la agenda de la Conferencia de Río de Janeiro en 1992 de la que se derivaron la creación de las Convenciones de Biodiversidad, de Desertificación y de Cambio Climático. En dicha Conferencia de 1992, hubo un planteamiento inicial basado en la responsabilidad de los países desarrollados en la generación de los problemas ambientales existentes y, por ende, la responsabilidad que tienen de brindar recursos financieros, tanto para llevar a cabo sus propias acciones, como para apoyar la acción de países en desarrollo.

En este sentido, la creación de la Convención Marco de Naciones Unidas sobre Cambio Climático (CMNUCC), fue la que estableció la relación donante-receptor que perduró por años. El Artículo $3^{\circ}$ de la CMNUCC apuntala que "las Partes que son países desarrollados y las demás Partes desarrolladas que figuran en el anexo II, proporcionarán recursos financieros nuevos y adicionales para cubrir la totalidad de los gastos convenidos que efectúen las Partes que son países en desarrollo para cumplir sus obligaciones" (CMNUCC, Artículo 3).

Al respecto, la Convención señala en su Artículo $5^{\circ}$ que "las Partes que son países en desarrollo y las demás Partes desarrolladas que figuran en el anexo II tomarán todas las medidas posibles para promover, facilitar y financiar, la transferencia de tecnologías y conocimientos prácticos ambientalmente sanos, o el acceso a ellos, a otras Partes, especialmente las Partes que son países en desarrollo, a fin de que puedan aplicar las disposiciones de la Convención" (CMNUCC, Artículo 5).

Este mandato es el que ha guiado de manera primaria la visión del financiamiento para atención del cambio climático, no obstante, su interpretación se ha dado en función de las necesidades de los países. Si bien, se reconoce que los países desarrollados son los que proporcionarán recursos, el Artículo 5 señala que los países en desarrollo deben promover, facilitar y financiar también tecnologías y conocimiento para atender el problema.

Aunque hay un manifiesto de que las diversas partes deben tomar medidas, ha existido discrepancia en la interpretación de esto. Por una parte, dentro del grupo de países en desarrollo, se encuentran diversos

Revista Política, Globalidad y Ciudadanía, Vol. 3 No. 5, Enero - Junio 2017, Universidad Autónoma de Nuevo León, Monterrey, México Monterrey, México, ISSN 2395-8448. pp 65-77. http://revpoliticas.uanl.mx/index.php/RPGyC/article/view/68 
países, como la mayoría de países africanos y los denominados países menos desarrollados, cuyas capacidades económicas son restringidas. Por otra parte, esta misma clasificación incluye a países como China, India, Brasil y otros, que se encuentran en una trayectoria de desarrollo y crecimiento económico distinta, y por tanto su incorporación dentro de esta clasificación ha sido puesta en tela de juicio, por considerarse economías de renta media que no deberían recibir más fondos de cooperación.

Este debate es el que perduró en el marco de las negociaciones de cambio climático durante años, hasta hace poco con la creación, aprobación y entrada en vigor del Acuerdo de París. El Acuerdo, si bien reconoce que los países desarrollados deben mantener el liderazgo en la provisión y movilización de flujos de financiamiento, también reconoce y da la bienvenida a que "otras partes" en capacidad de hacerlo, provean de financiamiento para la atención del cambio climático (Acuerdo de París, Artículo 9).

Lo anterior, ha traído nuevos debates a la esfera internacional, como la manera en que los países deben definir lo que es y lo que no es financiamiento climático y en qué momento dicho financiamiento debe ser o no contabilizado. Esto debido a que en 2009 se propuso y en 2010 se acordó, que los países desarrollados transferirán 100 mil millones de dólares anuales a países en desarrollo a partir de 2020 (COP15, Copenhague y COP16, México).

Aunque no existe un sustento que justifique la cifra de los 100 mil millones, es la cifra que ha guiado el debate internacional, y el mayor cuestionamiento ha sido la manera en que los países alcanzarán dicha transferencia y la importancia de definir las fuentes que serán parte de esta transferencia, es decir, si serán fuentes públicas o si también se contarán fuentes privadas.

Para ello, el Comité Permanente de Financiamiento llevó a cabo en 2014 su primera evaluación bianual de flujos de financiamiento de cambio climático, con el fin de conocer las cantidades transferidas para 2011 y 2012. En dicho estudio se observó que en 2011 se transfirieron 340 mil millones de dólares, mientras que en 2012 se transfirieron 650 mil millones (SCF, 2014).

En un segundo estudio publicado en 2016, el Comité identificó que en 2013 se transfirieron 687 mil millones de dólares y en 2014, 741 mil millones (SCF, 2016). Los resultados obtenidos en estos dos estudios muestran que las cifras son superiores a los 100 mil millones comprometidos en 2009; sin embargo, el debate se ha concentrado en la definición de lo que el Comité contempló en estas estimaciones y la necesidad de revisar que no se traten de cifras que hayan sido doblemente contadas.

En este sentido y derivado del Acuerdo de París, actualmente se encuentran en proceso de definición las modalidades de contabilidad de financiamiento provisto y movilizado. Si dichas modalidades son implementadas a cabalidad, esto permitirá definir con claridad qué será y qué no será contabilizado como financiamiento climático y, por ende, cuál es la cifra que realmente está fluyendo y con ello, identificar las brechas existentes entre lo que se tiene y lo que se necesita.

Tales modalidades podrían ayudar a definir lo que la comunidad internacional debería entender como financiamiento climático, ya que a la fecha no existe una definición universalmente aceptada.

La relevancia de esta discusión radica en que, mientras existe una creciente necesidad de actuar en materia de mitigación y adaptación, aún no existe una definición de qué tipo de actividades serán consideradas como mitigación o adaptación. Un ejemplo de esta discrepancia se presentó en 2015 cuando la Organización para la Cooperación y el Desarrollo Económico (OECD) y la Iniciativa de Política Climática (CPI), presentaron un informe sobre el estatus del financiamiento global de cara al cumplimiento de los 100 mil millones de dólares, y dentro de su metodología se determinó que ciertas actividades no deberían ser consideradas como cambio climático, aun cuando los donantes así lo consideren. Este fue el caso de proyectos relacionados con "carbón limpio" promovidos por países como Japón (OECD, CPI, 2015).

La importancia de la definición de financiamiento climático también radica en que en 2015 los países parte de la CMNUCC, fueron llamados a someter sus contribuciones nacionalmente determinadas (NDCs). Éstas son acciones que los países, tanto desarrollados como en desarrollo, proponen poner en marcha para cumplir con el Acuerdo de París y así evitar un aumento de la temperatura global de más de $2^{\circ} \mathrm{C}$ e idealmente en no más de $1.5^{\circ} \mathrm{C}$.

Dichas contribuciones son de carácter nacional, es decir, que fueron definidas por países partes y presentan una serie de propuestas y medidas que están dispuestos a implementar como nación. Algunos países

Revista Política, Globalidad y Ciudadanía, Vol. 3 No. 5, Enero - Junio 2017, Universidad Autónoma de Nuevo León, Monterrey,

México Monterrey, México, ISSN 2395-8448. pp 65-77. http://revpoliticas.uanl.mx/index.php/RPGyC/article/view/68 
sometieron contribuciones que los países están dispuestos a implementar de manera incondicional, es decir, con sus propios recursos, mientras que la mayoría de los países presentaron contribuciones condicionadas a la existencia de apoyo financiero (CMNUCC, 2015).

En este sentido, la importancia de definir qué es el financiamiento climático, será un factor que ayudará a definir cuáles de las acciones integradas por los países serán susceptibles de financiamiento internacional o no. Esto es particularmente relevante para una región como América Latina que es altamente vulnerable pero también responsable del $9 \%$ de las emisiones globales (CEPAL).

\section{El financiamiento climático en América Latina}

Estudios realizados por organismos como el Overseas Development Institute (ODI) y la Fundación Heinrich Böll, muestran que la región de América Latina y el Caribe no es la principal receptora de financiamiento climático y que la región de Asia Pacífico es la que recibe la mayor cantidad de fondos en ese rubro. Por otro lado, del financiamiento climático recibido en Latinoamérica, se ha comprobado que existe una inequitativa distribución de los fondos. Históricamente Brasil y México han sido los mayores receptores del financiamiento climático de la región (55\%), habiendo conseguido entre ambos países más de 1,500 millones de dólares entre 2003 y 2016. (ODI, HBF, 2016). Este financiamiento se refiere principalmente a fondos climáticos como el fondo de adaptación, el Fondo Verde del Clima, y otros en operación bajo la CMNUCC.

De igual forma, se ha identificado que la mayor parte del financiamiento se dirige a acciones de mitigación, pese a ser una región altamente vulnerable y requerir atención específica en el tema de adaptación al cambio climático. Esta tendencia se confirma también con el estudio del Comité, ya citado, que ha detectado que $70 \%$ del financiamiento climático se va a mitigación y sólo $25 \%$ a la adaptación.

Por otro lado, de acuerdo a un estudio llevado a cabo por el Grupo de Financiamiento Climático para Latinoamérica y el Caribe (GFLAC), se identifica que la mayor parte de los países de la región hacen mención sobre la necesidad de tener recursos financieros para cumplir con sus compromisos. Sin embargo, el estudio muestra que pocos países han integrado cifras sobre las necesidades de financiamiento y estrategias claras de cómo desean implementar dichas contribuciones (GFLAC, 2015).

El estudio señala que pese a reconocer la necesidad del financiamiento, aún existen limitaciones en la identificación e integración del tema de financiamiento como eje transversal en las políticas climáticas.

En la revisión de casos, el GFLAC identificó que los dos principales instrumentos que se han utilizado para la provisión de financiamiento internacional son préstamos y donaciones (Estudios de GFLAC en Argentina, Bolivia, Chile, Ecuador, Guatemala, Honduras, Nicaragua, y Perú, 2014-2015).

Tabla 1. Flujos internacionales de financiamiento climático en ocho países latinoamericanos (en millones de dólares)

\begin{tabular}{|c|c|c|c|c|c|c|c|}
\hline País & $\begin{array}{c}\text { Periodo } \\
\text { analizado }\end{array}$ & $\begin{array}{c}\text { Total } \\
\text { financiamiento } \\
\text { climático } \\
\text { recibido } \\
\text { (millones de } \\
\text { dólares USD) }\end{array}$ & Préstamo & Donaciones & Adaptación & Mitigación & Otro \\
\hline Argentina & $2010-14$ & 283 & 164 & 118 & 127 & 66 & 90 \\
\hline Bolivia & $2010-14$ & 318 & & & 261 & 6 & 52 \\
\hline Chile & $2010-14$ & 304 & 203 & 101 & 15 & 287 & \\
\hline Ecuador & $2010-14$ & 2,223 & 1,959 & 228 & 362 & 830 & 1,030 \\
\hline Guatemala & $2010-15$ & 338 & 237 & 101 & 38 & 30 & 268 \\
\hline Honduras & $2010-15$ & 227 & 79 & 148 & 62 & 91 & 74 \\
\hline Nicaragua & $2010-15$ & 322 & 131 & 192 & & & \\
\hline Perú & $2010-13$ & 1,554 & 1,159 & 396 & 498 & 761 & 296 \\
\hline \multirow[t]{2}{*}{ Total } & & 5,570 & 3,931 & 1,284 & 1,364 & 2,071 & 1,809 \\
\hline & & & $74.9 \%$ & $24.2 \%$ & $26.0 \%$ & $39.5 \%$ & $34.5 \%$ \\
\hline
\end{tabular}

Fuente: Elaboración propia, 2017.

Revista Política, Globalidad y Ciudadanía, Vol. 3 No. 5, Enero - Junio 2017, Universidad Autónoma de Nuevo León, Monterrey, México Monterrey, México, ISSN 2395-8448. pp 65-77. http://revpoliticas.uanl.mx/index.php/RPGyC/article/view/68 
Un aspecto fundamental en este sentido es la visión de financiamiento que se ha creado a nivel internacional, la cual está centrada en la cooperación internacional, cuando existen una diversidad de mecanismos financieros que pueden ser parte de la provisión de recursos para la atención del problema.

En este sentido, el GFLAC también propone la revisión no sólo de los flujos que se reciben de cooperación internacional, sino también aquellos que se asignan vía los presupuestos públicos. En estudios realizados por la organización sobre gasto público en materia de cambio climático se ha encontrado que, si bien el gasto relacionado con cambio climático se ha incrementado, en general los países destinan menos del $1 \%$ de su presupuesto público a financiar acciones de cambio climático, con la excepción de Guatemala, Honduras y Nicaragua (GFLAC, 2017).

Tabla 2. Gasto público nacional relacionado con el cambio climático en América Latina (siete países)

\begin{tabular}{|c|c|c|c|}
\hline País & Año & $\begin{array}{l}\text { Gasto climático } \\
\text { (millones de } \\
\text { dól ares USD) }\end{array}$ & $\begin{array}{l}\% \text { del presupuesto } \\
\text { nacional total }\end{array}$ \\
\hline \multirow[t]{4}{*}{ ARGENTINA } & 2013 & 134 & $0.12 \%$ \\
\hline & 2014 & 119 & $0.11 \%$ \\
\hline & 2015 & 81 & $0.06 \%$ \\
\hline & 2016 & 139 & $0.09 \%$ \\
\hline \multirow[t]{2}{*}{ BOLIVIA } & 2014 & 252 & $0.89 \%$ \\
\hline & 2015 & 258 & $0.80 \%$ \\
\hline \multirow[t]{2}{*}{ CHILE } & 2013 & 16 & $0.03 \%$ \\
\hline & 2014 & 21 & $0.04 \%$ \\
\hline \multirow[t]{2}{*}{ GUATEMALA } & 2014 & 191 & $2.45 \%$ \\
\hline & 2015 & 233 & $2.94 \%$ \\
\hline \multirow[t]{2}{*}{ HONDURAS } & 2014 & 174 & $1.89 \%$ \\
\hline & 2015 & 184 & $2.15 \%$ \\
\hline \multirow[t]{2}{*}{ NICARAGUA } & 2014 & 23 & $1.04 \%$ \\
\hline & 2015 & 26 & $1.13 \%$ \\
\hline \multirow[t]{2}{*}{ PERÚ } & 2013 & 82 & $0.22 \%$ \\
\hline & 2014 & 112 & $0.28 \%$ \\
\hline
\end{tabular}

Fuente: Elaboración propia, 2017.

\section{Financiamiento para la transición energética en Latinoamérica}

En América Latina y el Caribe, la principal fuente de emisiones es el sector energético que representa el $42 \%$ de las emisiones totales de la región (CEPAL, 2015b). Esto resulta particularmente relevante ya que se espera que la demanda de energía primaria de la región sea al menos $80 \%$ más alta que los niveles actuales (IDB, 2016), significando así un enorme riesgo de aumento de emisiones en la región. Para hacer frente a la creciente demanda energética de Latinoamérica a la vez que se mantengan los compromisos climáticos, será entonces de suma importancia tener una oferta de energía baja en emisiones y por las condiciones de la región, la opción que parece óptima son las energías renovables.

Latinoamérica es una región altamente favorecida por la naturaleza: cuenta con radiación solar muy alta, fuertes vientos en diversas zonas, corrientes de agua vastas, yacimientos geotérmicos, etc. El potencial de la región se ha ido aprovechando cada vez más y se ve reflejado en la creciente inversión en energías renovables que fue de 4.6 miles de millones de dólares en 2005 y aumentó drásticamente a 16.4 miles de millones de dólares en 2015.

Costa Rica, por ejemplo, cuenta con una oferta eléctrica más limpia que países como Estados Unidos o Australia. El país tico genera más del $99 \%$ de su electricidad a través de 5 fuentes renovables: hidroeléctrica (78\%), eólica (10\%), geotermia (10\%), biomasa y solar (1\%) y en noviembre de 2017 rompió su propio record al abastecer su demanda eléctrica exclusivamente por renovables durante 300 días del año. 
De 2005 a 2009, Brasil fue el país que atrajo el mayor monto de inversiones en renovables en la región (70\%), sin embargo, desde 2010 esa diferencia ha ido a la baja y cada vez más, la inversión en renovables se distribuye en más países y a través de la región (ver Imagen 1). En 2015 la inversión en renovables destinada a Brasil fue del $40 \%$.

Figura 1. Inversión en energía renovable en Latinoamérica por país

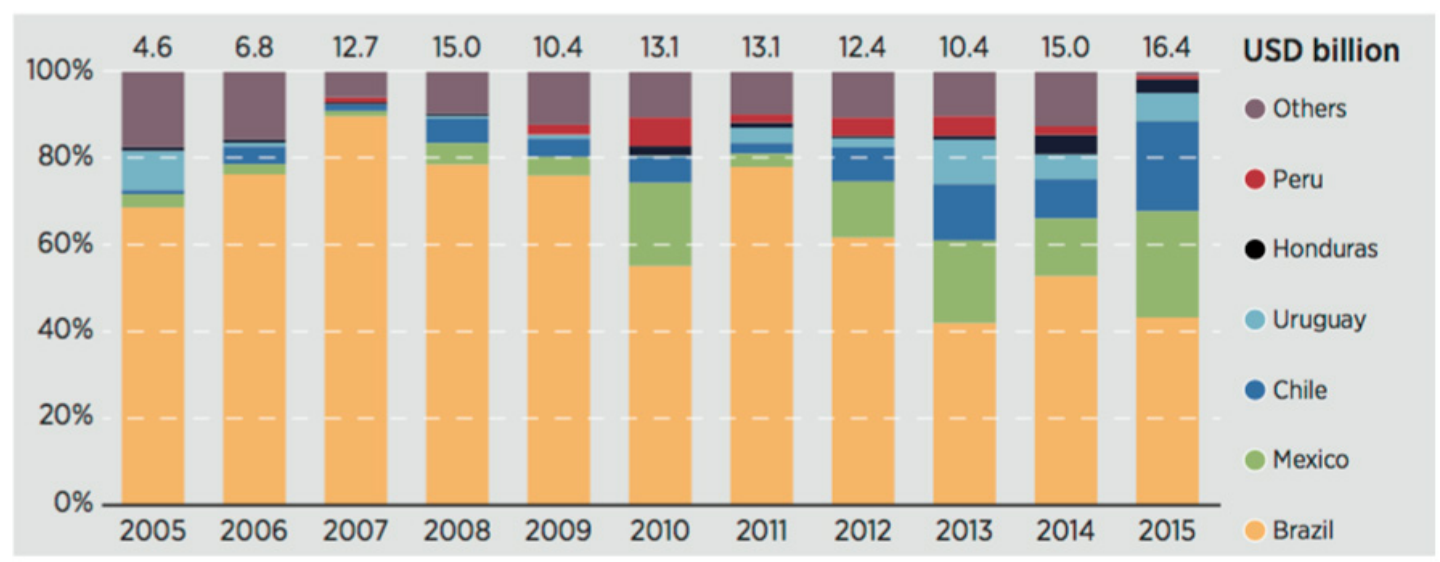

Fuente: IRENA, (2016).

Cabe señalar que el sector energético en donde más se ha invertido ha sido el eléctrico y cada vez más se ha dejado de lado a los biocombustibles. Por otro lado, es necesario también identificar que las inversiones corresponden a un incremento en la capacidad instalada eléctrica y la producción de electricidad renovable (ver Imágenes 2 y 3). No basta con invertir mucho (o poco), pues la inversión va asociada al precio de la tecnología en la que se invierte, es decir que, para medir la efectividad de la inversión en renovables, la capacidad instalada y generación eléctrica deben observarse también al alza.

Figura 2. Capacidad eléctrica renovable (excluyendo grandes hidroeléctricas

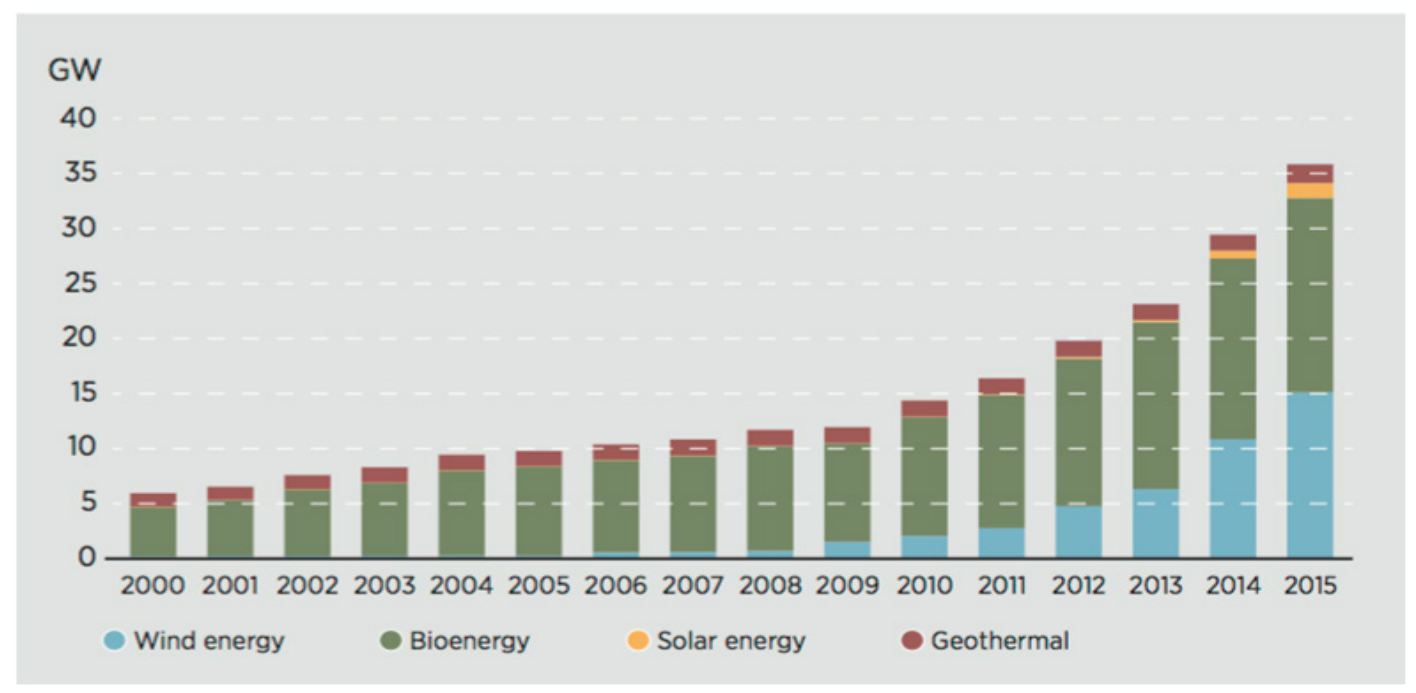

Fuente: IRENA, (2016). 
Figura 3. Generación eléctrica renovable (excluyendo grandes hidroeléctricas)

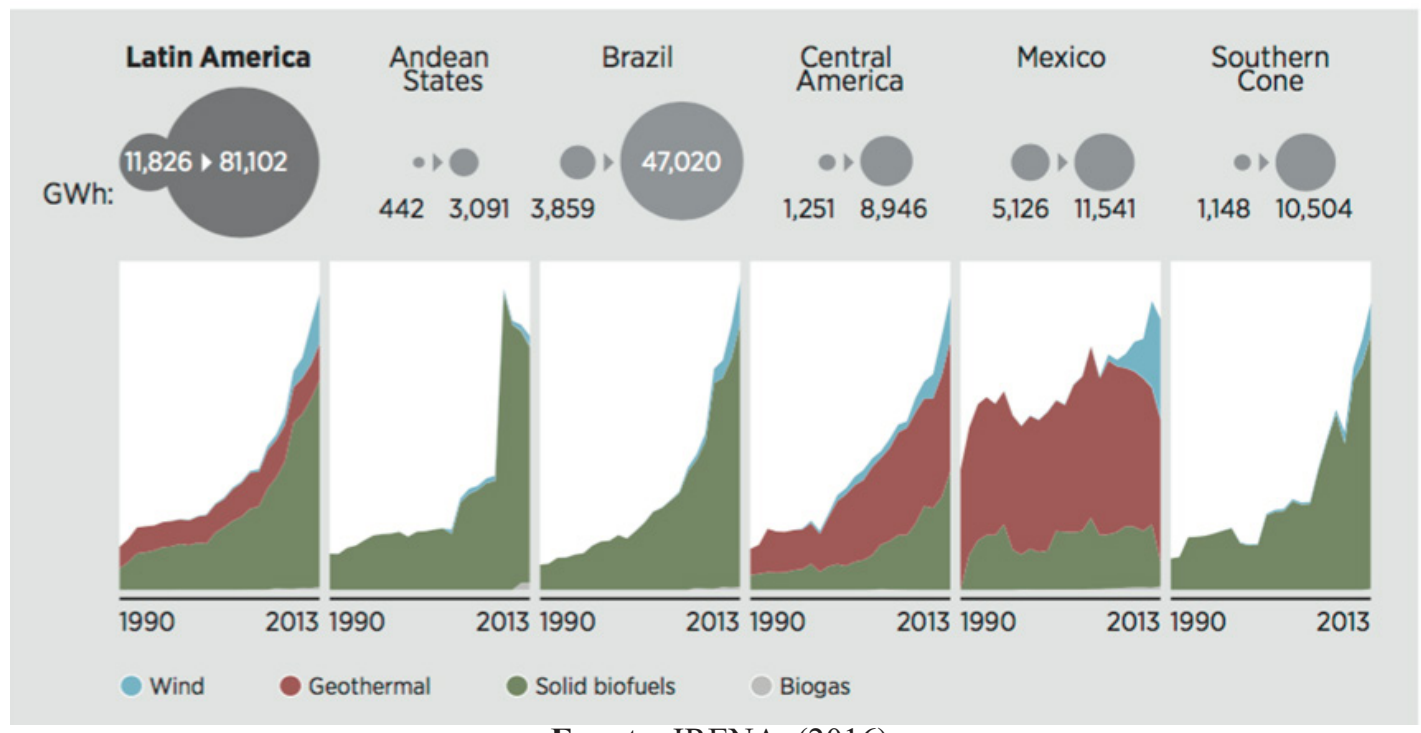

Fuente: IRENA, (2016).

Latinoamérica tiene las emisiones de carbono más bajas por la generación de electricidad, sin embargo, todavía existen 15 millones de personas en la región sin acceso a electricidad. El reto será entonces proveer esa necesidad eléctrica a través de fuentes renovables y, sobre todo, que la mezcla energética total de la región tenga una mucho mayor participación de renovables (ver Imagen 4).

Figura 4. Total de suministro de energía primaria en Latinoamérica en 2013

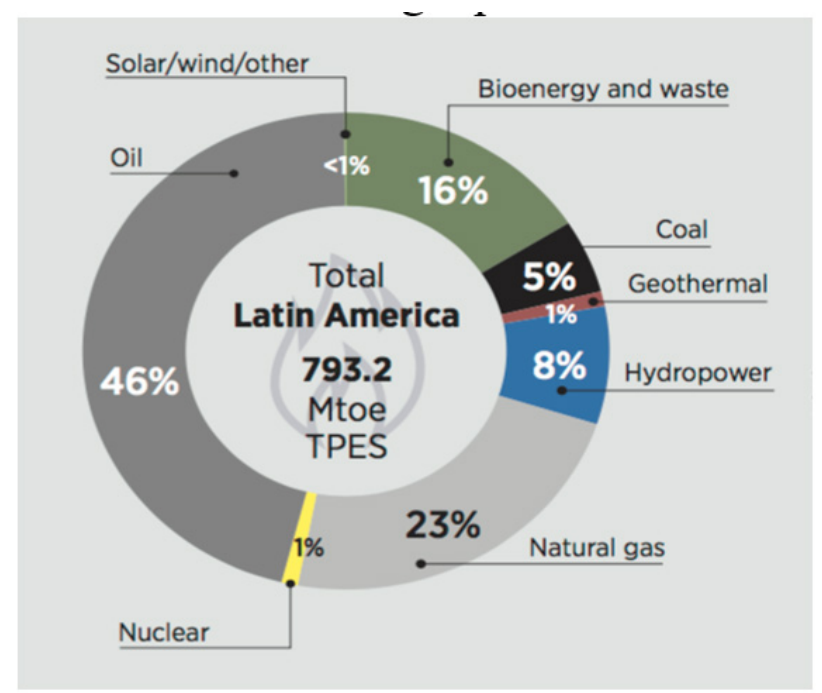

Fuente: IRENA, (2016).

Se espera que el pico de emisiones mundial por la generación de energía se dé en 2050, mientras que, de acuerdo con el reporte New Energy Outlook 2017, el pico de emisiones por la producción de electricidad se dará mucho antes, en 2026. Esto debido al retiro de emisiones de China por la generación eléctrica a través 
de carbón. El New Energy Outlook 2017 provee también un pronóstico alentador para México, al establecer que para 2040 el país generará el $80 \%$ de su electricidad a través de renovables (la energía solar se espera que tome un rol más importante y el país se convierta $29 \%$ más eficiente en el uso de electricidad). Sin embargo, el reporte también hace referencia a que la tasa de crecimiento de renovables no será suficiente para cumplir con los objetivos de reducción de emisiones globales.

A pesar de que 2016 vio un decremento del 23\% respecto a 2015 en la inversión mundial en energías renovables, hoy en día atestiguamos cifras alentadoras que indican que Latinoamérica va por un buen camino. El desarrollo de renovables se ha enfrentado a estructuras políticas y de instituciones fuertemente basadas en los combustibles fósiles en Latinoamérica (por ejemplo, México, Brasil y Venezuela), sin embargo, poco a poco las renovables han despegado y el propio mercado ha demostrado que hacen sentido económico para la región. Por ejemplo, en 2016 Honduras proveyó el 9.8\% de su electricidad a través de solar fotovoltaica, mientras que en Uruguay la energía eólica abasteció el 22.8\% de la demanda eléctrica. Por su parte, varias islas caribeñas como Aruba, Curazao, Bonaire y San Eustaquio, alcanzaron niveles de energía renovable superiores al $10 \%$ de su mezcla total.

En el mundo, se ha observado que, a pesar de las tendencias positivas, la tasa de desarrollo de renovables no será suficiente para lograr los compromisos del Acuerdo de París. Es necesario entonces acelerar el desarrollo de renovables y para ello se necesitan mitigar las barreras existentes que en cada caso les impiden despegar. Se han identificado dos aspectos clave para poder atraer inversiones privadas, ya sean locales o internacionales: 1) el acceso al financiamiento de los proyectos y de la infraestructura asociada, y 2) el compromiso y credibilidad de las entidades públicas a través de un marco legal específico, transparente y constante en el tiempo (CEPAL, 2012).

Será importante también implementar medidas de incentivación de renovables no sólo en el desarrollo de electricidad renovable, sino de energía renovable en su totalidad (calor, combustibles, etc.). Por otro lado, para poder incrementar la participación de renovables de manera sustentable, será clave adoptar medidas de eficiencia energética mucho más agresivas para disminuir la energía "fósil" que actualmente no se aprovecha.

Es un hecho que hay condiciones alentadoras en la región latinoamericana para el desarrollo de energías renovables, sin embargo, es necesario fortalecer este ímpetu con políticas claras y condiciones favorables para los inversionistas. Latinoamérica cuenta con el potencial natural, pero falta asegurar las demás condiciones para convertirlo en líder mundial.

\section{Presupuesto público en materia de cambio climático en México}

A nivel internacional se ha hablado mucho de la importancia de transferir recursos financieros para invertir en diversas actividades en materia de mitigación y adaptación y esta discusión se ha centrado mucho en la transferencia de fondos de países desarrollados a países en desarrollo. Sin embargo, el llamado del Acuerdo de París abre una nueva discusión que es la participación del financiamiento que viene de "otros países" que también están haciendo contribuciones, esto se refiere a países en desarrollo.

El papel que los países en desarrollo tendrán en el financiamiento climático también será crucial, y esto se relaciona con el rol de sus presupuestos públicos. El caso de México es significativo, pues es el segundo país emisor en la región. Estudios en progreso muestran la evolución del gasto público y lo limitado que ha sido la participación del mismo en las acciones de política climática (Guzmán, 2018).

En 2008 la partida presupuestal para el Programa Especial de Cambio Climático no existía, en 2009 se creó y se le asignaron recursos, mismos que en 2010 y 2011, en el contexto de la COP16 que se llevó a cabo en Cancún en diciembre de 2010, aumentó. Sin embargo, en 2012 dicho presupuesto tuvo una caída del $62 \%$, y para 2013 dicha partida presupuestal desapareció.

En el mismo año, se creó el Anexo 16 para acciones de mitigación y adaptación al cambio climático que en 2013 tuvo un ingreso de $\$ 3,956$ millones de pesos mexicanos (mdp). Esta cantidad se ha aumentado de manera muy limitada desde entonces, llegando a recibir $\$ 4,746$ mdp en 2016 que sólo representa el $0.9 \%$ del total del presupuesto.

Revista Política, Globalidad y Ciudadanía, Vol. 3 No. 5, Enero - Junio 2017, Universidad Autónoma de Nuevo León, Monterrey, México Monterrey, México, ISSN 2395-8448. pp 65-77. http://revpoliticas.uanl.mx/index.php/RPGyC/article/view/68 
El objetivo del Anexo es identificar el monto del gasto público asignado para hacer frente al cambio climático en las entidades relacionadas con el problema, en este caso los miembros de la Comisión Intersecretarial de Cambio Climático (CICC) (Guzmán, S, 2018). De acuerdo con Guzmán, la cantidad de dinero identificada desde 2013 hasta 2017, respectivamente, ha estado entre $\$ 34,514$ y $\$ 36,878 \mathrm{mdp}$, con la cantidad más baja en 2017 (2018).

Figura 5. Evolución de la asignación presupuestaria al Anexo sobre cambio climático.

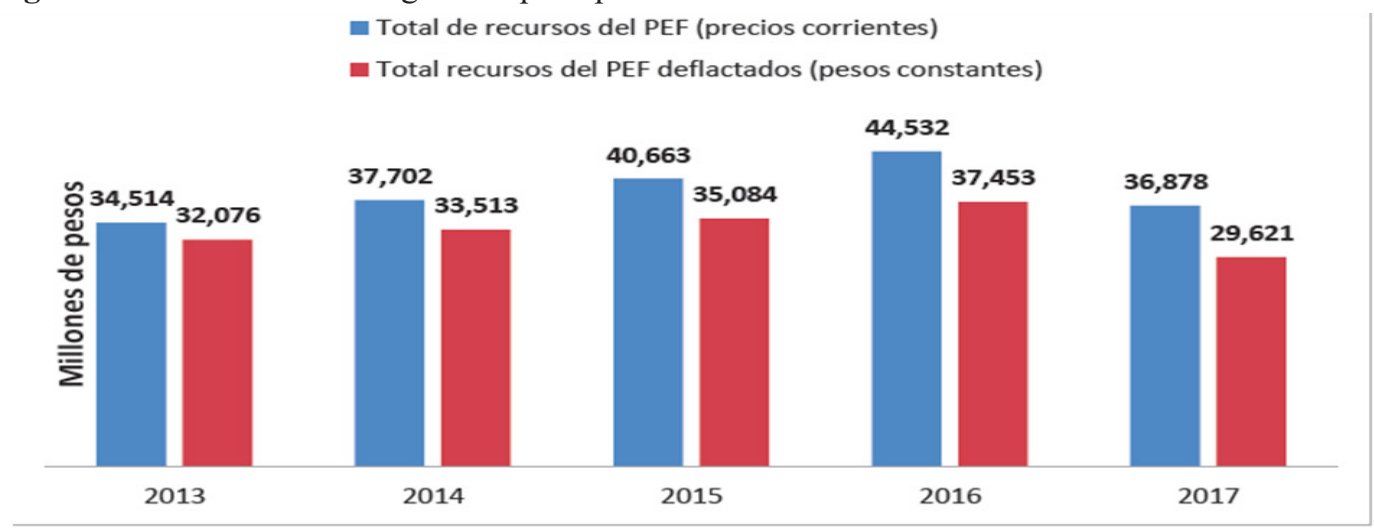

Fuente: INECC, (2017).

En 2017, la Oficina de Evaluación del Instituto Nacional de Ecología y Cambio Climático (INECC), que se encarga de evaluar el progreso de la política climática en México de acuerdo con la Ley General de Cambio Climático (LGCC), llevó a cabo una evaluación sobre el funcionamiento de este Anexo. Con base en esta evaluación, se identificaron tres aspectos clave, entre otros:

1. No existe una metodología para definir claramente qué constituye actividades de cambio climático. Se identificó que las personas que organizan la información presupuestaria no son las mismas que quienes están a cargo del diseño real de los programas. En ese sentido, no existe una correlación entre los objetivos de la política y la asignación del presupuesto público.Si bien la Secretaría de Hacienda y Crédito Público (SHCP) está a cargo de la provisión de metodologías para la creación de estos Anexos, la metodología que ellos brindan no incluye la consideración o los criterios del cambio climático para definir lo que cuenta como cambio climático. Sólo incluye disposiciones relacionadas con la presentación de la información presupuestaria.

2. No existe una correlación entre lo que se incluyó en el Anexo y las medidas incluidas en el Programa Especial de Cambio Climático (PECC), que según la Ley es el documento que debe definir las disposiciones presupuestarias para implementar las actividades incluidas en el programa. Esto significa que no todos los programas incluidos en el Anexo son parte del PECC, y otros programas incluidos en el PECC no son parte del Anexo. El informe del INECC señala que los servidores públicos entrevistados expresaron que no toman en cuenta sus compromisos establecidos en el PECC, pero que la prioridad para las dependencias es el cumplimiento de sus objetivos sectoriales (2017: 27) que van más allá del cambio climático.

3. El Sistema Nacional de Cambio Climático (SINACC) y la CICC, que son los dos organismos con atribuciones para definir la orientación para incorporar el cambio climático dentro de los procesos de planificación y particularmente para el cumplimiento de los objetivos incluidos en el PECC, no han proporcionado orientación para incorporar el cambio climático dentro del gasto público, y por lo tanto, tal ejercicio aún no se ha integrado en el Anexo. En la misma línea, se identificó que el papel del Congreso como entidad con la atribución de aprobar el presupuesto, no ha jugado un papel en el proceso de integración del cambio climático en la aprobación del presupuesto cada año. 
El presupuesto público en general ha sufrido recortes, sin embargo, los recortes más importantes se han dado en el sector ambiental. Por ejemplo, la propuesta de Presupuestos de Egresos de la Federación (PEF) 2017 para medio ambiente presenta una reducción del 47\% con respecto a lo aprobado en 2016.

La asignación de recursos para la atención al cambio climático, se vincula también con la protección de derechos humanos fundamentales, consagrados en la Constitución Mexicana. En este sentido, existen sub-funciones dentro del ramo de medio ambiente que son claves para la protección de los derechos humanos de las y los mexicanos. El caso de la sub-función "Administración del Agua" presenta una reducción de más de $\$ 1,700$ mdp, lo cual significa una caída real de 25.68\%. Mientras que la sub-función "Ordenación de aguas residuales, drenaje y alcantarillado" tiene un recorte de $\$ 3,802 \mathrm{mdp}$, lo que en términos reales se traduce en una reducción de 31.3\%. Más aún, sub-funciones como "reducción de la contaminación" y "otros temas de Protección Ambiental", muestran un recorte de $\$ 340$ mdp, es decir, una caída real de $25.29 \%$, y la segunda de $\$ 17,016 \mathrm{mdp}$, lo que representa, en términos reales, una reducción del $63.27 \%$ (PAN, 2016).

Esto no sólo es un problema para el sector en general, sino para temas de alta relevancia para el país como el cambio climático. En el caso del presupuesto para cambio climático, aunque la tendencia de la asignación presupuestal ha mostrado algunas mejoras en recientes años, ésta no es suficiente.

Lo anterior quiere decir que, aunque el tema de cambio climático ya no es sólo uno de tipo ambiental, sino de tipo transversal que requiere de la participación de más sectores y que además afectará a otros tantos, el país sólo gasta $0.9 \%$ de su presupuesto en atender este tema. No obstante, México se ha colocado como líder en la agenda internacional de cambio climático y desde el GFLAC señalamos que no existe congruencia entre las acciones y compromisos establecidos a nivel internacional y las acciones que el país realiza a nivel nacional.

\section{3.- MÉTODO}

\section{Diseño}

El enfoque investigativo de la presente investigación es cualitativo, de acuerdo con Hernández, Batista y Fernández (2014) "Utiliza la recolección y análisis de los datos para afinar las preguntas de investigación o revelar nuevas interrogantes en el proceso de interpretación” (p.7).

Alcanzando un diseño no experimental "Que se realiza sin la manipulación deliberada de variables y en los que sólo se observan los fenómenos en su ambiente natural para después analizarlos" (Hernández, Batista y Fernández, 2014, p. 149).

El alcance establecido es el exploratorio "emplean cuando el objetivo consiste en examinar un tema poco estudiado o novedoso" (Hernández, Batista y Fernández, 2014, p. 91).

\section{Instrumentos}

Para la construcción del marco teórico-conceptual del financiamiento de esfuerzos contra el cambio climático, se consultaron un total de treinta y ocho referencias bibliográficas utilizándose como instrumento las ideas, argumentos y proyectos que fueron interpretados desde una perspectiva analítica y crítica.

\section{Procedimiento}

Con relación a la comprensión del problema de la investigación se recopilan fuentes secundarias de documentos académicos. En el marco de referencia se definen los conceptos básicos relativos al financiamiento al esfuerzo contra el cambio climático. Una vez recopilada y analizada la información se construye el documento objeto de este trabajo. Por último, se realizan las recomendaciones y conclusiones conforme a los objetivos trazados (Bascón et al, 2016, p. 39). 


\section{CONCLUSIONES}

Estado será clave en el desarrollo de renovables, pues se requieren mitigar aceleradamente las barreras que impiden su desarrollo para poder asegurar más inversiones en la región que se den de manera Cada vez es más evidente que, para alcanzar los objetivos que se establecieron en el Acuerdo de París, será necesaria una acción conjunta y determinante de todos los actores involucrados. Los gobiernos nacionales y locales, el sector privado, los fondos multilaterales, la banca, la sociedad civil, etc. deberán coordinar en conjunto y eficazmente las acciones que nos permitan mantener a nuestro planeta por debajo de un aumento de temperatura de $1.5^{\circ} \mathrm{C}$.

Para lo anterior, se requiere que los países establezcan de manera clara los costos para alcanzar las metas establecidas en sus contribuciones nacionales, así como la identificación de los recursos que actualmente reciben y hacia donde se están destinando estos recursos, para verificar que efectivamente están alineados con las prioridades establecidas a nivel nacional en materia de cambio climático.

Como se presentó en este artículo, es necesario contar con metodologías para contabilizar el financiamiento climático, en las que se establezca qué actividades van a ser consideradas como financiamientos climáticos y cuáles no. Contar con información precisa sobre el financiamiento climático, es fundamental para apoyar la toma de decisiones tanto a nivel internacional como nacional, de modo que los actores relevantes puedan reconocer dónde hay vacíos en la provisión de financiamiento y orientar efectivamente sus esfuerzos para solventar esas deficiencias.

A pesar de las diferencias metodológicas que se traducen en cifras distintas, la información existente muestra que la mayor parte de los recursos se destinan a acciones de mitigación, requiriendo mayores esfuerzos para dirigir recursos para reducir vulnerabilidad e incrementar resiliencia. En cuanto a mitigación, hablando particularmente del sector energético de Latinoamérica, la inversión en energías renovables es una clara medida que se deberá de tomar para evitar que la región aumente desmesuradamente sus emisiones.

\section{REFERENCIAS}

African Development Bank (AfDB), Asian Development Bank (ADB), European Bank for Reconstruction and Development (EBRD), European Investment Bank (EIB), Inter-American Development Bank (IDB), and the International Finance Corporation (IFC) and the World Bank (WB) from the World Bank Group (WBG), (2014) Joint Report on Multilateral Developments Banks'Climate Finance, June 2015.

African Development Bank (AfDB), Asian Development Bank (ADB), European Bank for Reconstruction and Development (EBRD), European Investment Bank (EIB), Inter-American Development Bank Group (IDBG), and World Bank Group (WBG) (2015). Joint Report on Multilateral Developments Banks' Climate Finance, August 2016.

Aguilar, S. y Scardamaglia, V. (2014), Financiamiento Internacional para el Cambio Climático en Argentina, FLACSO Argentina- GFLAC.

Bascón, M.: Cazallo, A.; Lechuga, J. \& Meñaca, I. (2016). Estudio de la necesidad de implantar un servicio público de transporte entre las ciudades de Ceuta-Tetuán y Melilla-Nador. En Desarrollo Gerencial Revista de la Facultad de Ciencias Económicas, administrativas y contables de la Universidad Simón Bolívar 8(2), $37-57$.

Balza, L. H., Espinasa, R., y Serebrisky, T. (2016). Lights on? energy needs in Latin America and the Caribbean to 2040, Inter-American Development Bank (IDB).

Barnard, S., Watson, C. y Schalatek, L. (2016). Climate Finance Regional Briefing: Latin America Climate Finance Fundamentals 6, Overseas Development Institute (ODI) \& Heinrich Böll Stiftung North America (HBS).

Revista Política, Globalidad y Ciudadanía, Vol. 3 No. 5, Enero - Junio 2017, Universidad Autónoma de Nuevo León, Monterrey, México Monterrey, México, ISSN 2395-8448. pp 65-77. http://revpoliticas.uanl.mx/index.php/RPGyC/article/view/68 
Bloomberg New Energy Finance (2017), New Energy Outlook 2017, BNEF.

Bloomberg (2017). Latin America's clean-power play. https://www.bloomberg.com/view/ articles/2017-05-17/latin-america-s-clean-power-play

Coviello, M.F., Gollan, J., y Perez, M. (2012). Las alianzas público-privadas en energías renovables en América Latina y el Caribe, Comisión Económica Para América Latina y el Caribe (CEPAL).

DNP (Departamento Nacional de Planeación) (2016), Guia para medir actividades asociadas a la mitigación y a la adaptación del cambio climático en Colombia.

GFLAC (Grupo de Financiamiento Climático para América Latina y el Caribe) (2014), Handbook for the analysis of international financing and public budgets in the field of climate change in Latin America and the Caribbean, GFLAC.

Guzmán, S., Moncada, A., Canales, N., Castillo, M., y Guillen, T. - GFLAC (2017), “Toward Climate Finance Reporting Systems in Latin America", en Adaptation Watch, Toward Implementation: The 2017 Adaptation Watch Report.

Guzmán, S, Guillen, T, Manda, J. (2017). A Review of Domestic Data Sources for Climate Finance Flows in Recipient Countries. Colombia, Guatemala, Kenya, Nepal, The Philipines, and Zambia. UNDPGFLAC

Heinrich Böll Stiftung North America, Climate Funds Update (2017). Funding by region: Latin America. http://www.climatefundsupdate.org/regions/latin-america. (Last accessed: September 2, 2017)

Hernández, R., Fernández, C., y Baptista, P. (2014). Metodología de la investigación. México: Mc Graw Hill.

Hernández, J. (2014), Financiamiento Internacional para el cambio climático en Chile, Chile TransparenteGFLAC.

Huamani M., Suyana (2015). Financiamiento internacional para el cambio climático en Perú, DARGFLAC.

Independent UK (2017). Costa Rica's electricity generated by renewable energy for 300 days in 2017 (Ultima visita: 14 de enero de 2018) http://www.independent.co.uk/news/world/americas/costa-ricaelectricity-renewable-energy-300-days-2017-record-wind-hydro-solar-water-a8069111.html

Instituto Nacional de Ecología y Cambio Climático-INECC (2017). Informes de los resultados de la Evaluación Estratégica del Anexo Transversal y del Programa Especial de Cambio Climático. México.

International Renewable Energy Agency - IRENA (2016). Estadísticas de energía renovable 2016 América Latina y el Caribe, IRENA.

International Renewable Energy Agency - IRENA (2016). Renewable Energy Market Analysis: Latin America, IRENA, Abu Dhabi.

International Renewable Energy Agency - IRENA (2016). Latin America and the Caribbean. http://www. irena.org/lac (Última visita: 14 de enero de 2018).

Mairena-Arauz, D., Romero, R., y Méndez, C. (2015), Informe País de Financiamiento para el Cambio Climático, Nicaragua, CADPI -Centro Humboldt- GFLAC.

Organisation for Economic Co-operation and Development- OECD (2015). Climate finance in 2013-2014 and the USD 100 billion goal, OECD in collaboration with Climate Policy Initiative (CPI)

Paz C. (2015), Análisis de Flujos de Financiamiento Internacional de Cambio Climático. Estado Plurinacional de Bolivia, LIDEMA- GFLAC.

Revista Política, Globalidad y Ciudadanía, Vol. 3 No. 5, Enero - Junio 2017, Universidad Autónoma de Nuevo León, Monterrey, México Monterrey, México, ISSN 2395-8448. pp 65-77. http://revpoliticas.uanl.mx/index.php/RPGyC/article/view/68 
Partido Acción Nacional-PAN (2016). Presentación en foro "Por un presupuesto que Fortalezca la Sustentabilidad en México" llevado a cabo el 12 de octubre de 2016 en la Cámara de Diputados de México.

Peláez, M. y Herrera, J.J. (2014). Análisis de Financiamiento de Cambio Climático Internacional en Ecuador, Grupo Faro- GFLAC.

Pineda, C., Mejía, L. y Matute, L. (2015), Finanzas Internacionales en Cambio Climático, Honduras, AHCC -ACICAFOC-ANAFAE - GFLAC, Honduras, GFLAC.

REN21 (2017). Renewables 2017 Global Status Report, REN21 Secretariat, Paris.

Samaniego, J. y Schneider, H. (2015). Financiamiento para el cambio climático en América Latina en 2013, Comisión Económica Para América Latina y el Caribe (CEPAL).

Samaniego, J. (2015). The economics of climate change in Latin America and the Caribbean - Paradoxes and challenges of sustainable development, Comisión Económica Para América Latina y el Caribe (CEPAL).

Samaniego, J. y Schneider, H. (2016). Financiamiento para el cambio climático en América Latina en 2014, Comisión Económica Para América Latina y el Caribe (CEPAL).

Samaniego, J. y Schneider, H. (2017). Financiamiento para el cambio climático en América Latina en 2015, Comisión Económica Para América Latina y el Caribe (CEPAL).

Torselli, C. y Morataya, M. (2015). Financiamiento internacional para el cambio climático en Guatemala, Fundación Solar-GFLAC.

United Nations Framework Convention on Climate Change -UNFCCC (2016). 2016 Biennial Assessment and Overview of Climate Finance Flows Report, United Nations.

United Nations Framework Convention on Climate Change -UNFCCC (2017a). INDC Portal recuperado de: (http://www4.unfecc.int/submissions/inde/Submission\%20Pages/submissions.aspx)

United Nations Framework Convention on Climate Change -UNFCCC (2017b). Submitted Biennial Update Reports (BURS) from Non- Annex I Parties recuperado: (http://unfccc.int/national_reports/ nonannex_i_natcom/reporting_on_climate_change/items/8722.php)

United Nations Framework Convention on Climate Change-UNFCCC (2017c). Submitted National Communications from Non-Annex I Parties. Recuperado de: (http://unfccc.int/national_reports/nonannex_i_natcom/items/10124.php) 